\title{
Can Crohn's disease be diagnosed at laparotomy?
}

\author{
R J Butterworth, G T Williams, L E Hughes
}

\begin{abstract}
The typical macroscopic features of Crohn's disease have been well described and are widely regarded as sufficient to diagnose the disease at laparotomy. We report six patients undergoing laparotomy for symptomatic Crohn's disease, shown radiologically, who were found to have macroscopically normal small bowel despite careful examination of the bowel by an experienced surgeon. In four cases resection was deferred, but all subsequently deteriorated and required further surgery. Minor abnormalities found by balloon examination of the terminal ileum prompted resection in two further patients. Histology showed an unusually superficial distribution of inflammation, which could explain the negative findings at laparotomy. We conclude that normal laparotomy findings alone do not exclude a diagnosis of clinically important small bowel Crohn's disease. Crohn's disease should be considered in patients with persisting symptoms after negative laparotomy.
\end{abstract}

The macroscopic features of small bowel Crohn's disease have been well described. ${ }^{1-10}$ Recognition of these is said to be a reliable aid to diagnosis at laparotomy when previous investigation has failed to establish the diagnosis. ${ }^{6}$ The descriptions in published reports relate to well established disease, and we can find no discussion of the accuracy of laparotomy assessment in cases where the operative findings are less clear cut.

While it is not uncommon to discover Crohn's disease unexpectedly at laparotomy, the opposite clinical problem, where apparently normal bowel is found at laparotomy in patients with symptoms and radiological findings highly suggestive of Crohn's disease, is unusual and presents considerable management difficulties. We have encountered six such patients out of 175 patients undergoing laparotomy for small bowel Crohn's disease over 18 years. The laparotomy assessment in each case was performed by one of the authors (LEH). We can find no guidance in publications on how to deal with this problem.

\section{Case reports}

University Departments of Surgery and Pathology, University Hospital of Wales, Heath Park, Cardiff CF4 4XN R J Butterworth G T Williams L E Hughes Correspondence to: Professor L E Hughes. Accepted for publication 2 April 199
CASE 1

An 18 year old girl presented with an ischiorectal abscess, abdominal pain, and diarrhoea. Barium studies showed a possible narrowing in the terminal ileum suggestive of Crohn's disease. Emergency laparotomy was needed for small bowel obstruction four months later, when a large mass was found in the ileal mesentery. However, the ileum appeared normal on careful detailed inspection and palpation. As clearance of the mesenteric mass would have required radical bowel resection, no resection was undertaken. A biopsy specimen of an enlarged node showed non-specific inflammation.

Symptoms persisted and two years later an ileocaecectomy was performed for ileal Crohn's disease with fistulation into the sigmoid colon, confirmed histologically.

Five years later increasing bowel symptoms led to laparotomy when the terminal ileum was completely normal on inspection and palpation and the previous anastomosis remained widely patient. No resection was undertaken. Four years later persisting symptoms and our experience reported in this paper led to a further laparotomy, when three obvious strictures of the ileum were treated by stricturoplasty.

\section{CASE 2}

A 20 year old man presented with diarrhoea and abdominal pain, and one year later developed multiple perineal and buttock sinuses. Barium studies suggested Crohn's disease of terminal ileum and caecum, with a fistula from terminal ileum to buttock. At laparotomy the small and large bowel appeared normal, except that a short segment of ileum was stuck down to the opening of a fistula in the floor of the pelvis. The ileum was mobilised and opened at the site of the fistula, where the bowel wall including the mucosa appeared normal. The fistulous opening was excised locally and closed directly. An ileal biopsy specimen from the margin of the fistula closure showed granulomatous inflammation.

Seven months later the patient's buttock sinuses reactivated, and barium studies suggested active Crohn's disease of the terminal ileum. Surprisingly, repeat laparotomy showed a macroscopically normal terminal ileum, but macroscopic changes of Crohn's disease in the transverse and descending colon. No procedure was undertaken, and the patient was treated medically. All disease is quiescent at present.

\section{CASE 3}

A 54 year old woman underwent resection of a Crohn's disease stricture of the terminal ileum, confirmed histologically. Two years later symptoms and radiology suggested recurrent Crohn's disease. At laparotomy recurrence was found in the terminal ileum but the disease was not gross and the previous anastomosis was widely patent. As the symptoms seemed to be due to dense adhesions, no resection was performed. A lymph node biopsy specimen showed non-specific inflammation.

The patient's symptoms persisted and a laparotomy three years later showed persisting adhesions, but the macroscopic Crohn's disease 
had resolved completely. An ileal enterotomy was performed, and a finger passed up and down the bowel and through the anastomosis. The mucosa appeared normal, the bowel wall was not thickened, and the lumen was of normal diameter. A mucosal biopsy specimen showed normal histology.

Bowel symptoms continued intermittently and colonoscopy seven years later showed recurrent disease at the previous anastomosis. The patient's symptoms were controlled by a short course of steroids and she has since remained asymptomatic without further medication.

\section{CASE 4}

An obese 28 year old woman presented in February 1985 with an ischiorectal abscess and biopsy was suggestive of Crohn's disease. Over the next 10 months she complained of progressive right iliac fossa pain and diarrhoea. Repeated barium studies reported stricturing due to Crohn's disease of the terminal ileum. The patient had little response to medical treatment and tolerated steroids badly. At laparotomy (November 1985) the bowel appeared normal on inspection and palpation. The appendix was removed and a balloon catheter inserted through the appendix stump to assess the bowel wall, which was found to be of normal thickness, supple, and without a stricture. The balloon could easily be seen through the distended bowel wall. Histology of the appendix and a mesenteric lymph node showed no abnormality.

The patient's symptoms persisted, and in March 1988 colonoscopy showed Crohn's disease affecting the caecum and ascending and descending colon, confirmed histologically. Examination of ileal biopsy specimens obtained by passage of biopsy forceps through the ileocaecal valve confirmed ileal involvement.

Increasing symptoms led to a further laparotomy (February 1989), where severe stenosing Crohn's disease affected the terminal ileum but the colon was relatively unaffected. In view of the previous findings at colonoscopy, the colon and terminal ileum were removed and ileorectal anastomosis performed. Histology confirmed classical ileal Crohn's disease but the colon showed focal mucosal inflammation only.

\section{CASE 5}

A 31 year old woman presented with abdominal symptoms of one year's duration and large fleshy anal skin tags. Barium studies, a technetium labelled white cell scan, and colonoscopy showed typical terminal ileal Crohn's disease.

Fourteen months after presentation laparotomy was performed with a view to terminal ileal resection, but the bowel looked and felt normal On passing a balloon catheter the bowel wall thickness was normal, except at the ileocaecal valve itself, which would allow passage of a balloon diameter of $1 \mathrm{~cm}$ only. On the basis of our previous experience, an ileocaecectomy was performed. Histology (Figure) confirmed Crohn's disease, with granulomatous inflammation of the mucosa and submucosa of the bowel wall and superficial fissuring ulceration. A single epithelioid granuloma was found in a mesenteric lymph node. These appearances are considered to represent an unusually superficial distribution of Crohn's disease.

The patient was symptom free eight months after surgery.

\section{CASE 6}

A 25 year old woman underwent ileocaecal resection in 1977 for Crohn's disease. Symptoms recurred after 13 years, requiring emergency admission with small bowel obstruction. Conservative treatment led to improvement, when a small bowel enema was inconclusive. The patient again deteriorated, and at laparotomy the bowel appeared normal. On passage of a balloon catheter, the bowel wall consistency and thickness were again normal except for a slight narrowing of the previous anastomosis. It was difficult to know whether this was abnormal, but in view of the experience with the earlier cases, a further resection was performed. Examination of the specimen showed a $4 \mathrm{~mm}$ area of mucosal cobblestoning at the previous anastomosis. Histology of this area showed focal mucosal inflammation, superficial fissuring ulceration, and lymphoid aggregates of recurrent Crohn's disease. The pathological features were, as in case 5, confined to the mucosa and submucosa. Postoperatively the patient's symptoms resolved.

\section{Discussion}

The appearance of the bowel wall in Crohn's disease has been well described by many authors. Crohn refers to the 'thickened, soggy and oedematous' nature of the terminal ileum, with thickened mesentery containing hyperplastic lymph nodes. ${ }^{1}$ Weakley and Turnbull ${ }^{6}$ describe 'serositis,' enlarged lymph nodes, and 'fatwrapping' of the bowel. Other common features include fistulation, stricture, segmental distribution, ${ }^{259}$ and inflammatory masses. A number of authors have emphasised the importance of palpating the mesenteric aspect of the bowel. Tiny nodules resembling tubercles ${ }^{49}$ are sometimes present on the bowel wall. On opening the bowel, cobblestoning of the mucosa, thickening of the bowel wall, and narrowing of the lumen

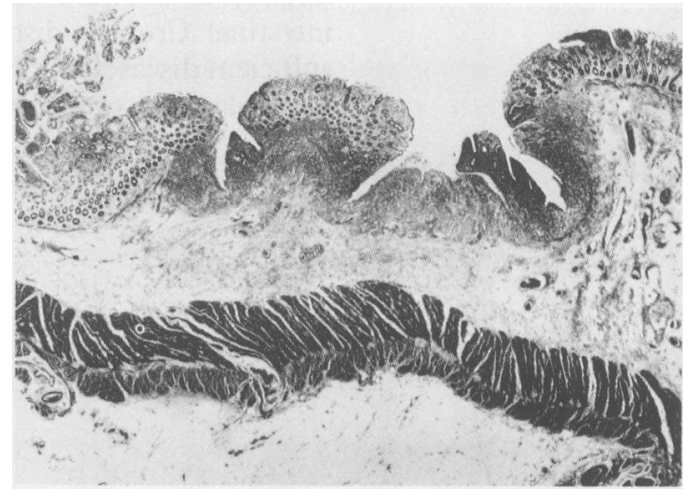

Crohn's disease confined to the mucosa and submucosa of the terminal ileum in case 5. There are early fissuring ulcers and the background mucosa showing variable degrees of villous atrophy and focal inflammation. Haemotoxylin and eosin (magnification $\times 16$ ). 
are said to be reliable features in the diagnosis of Crohn's disease. ${ }^{10}$

These macroscopic features are considered by many to be a valuable, and reliable, aid to diagnosis. Lockhart-Mummery and Morson ${ }^{78}$ maintain that 'at laparotomy the bowel usually appears obviously diseased,' and that 'it is usually possible to make a diagnosis of Crohn's disease on macroscopic evidence alone.' Weakley and Turnbull maintain that the signs described above are 'reliable visible signs in the diagnosis of regional ileitis, permitting early recognition, and that familiarity with them will enable establishing the diagnosis with confidence."

In all six of the reported cases the diagnosis of Crohn's disease was strongly suspected before laparotomy, but despite careful examination of the bowel, including enterotomy or balloon distension in four patients, none had any of the typical features of the disease. In cases where no bowel resection was performed because macroscopic appearances were normal (cases 1,3 , and 4), lymph node biopsy did not contribute to the diagnosis, even where nodes were enlarged. The reason for this is that while non-specific reactive hyperplasia of lymph nodes is common in Crohn's disease, specific granulomatous inflammation is not. ${ }^{11}$

A related question is whether macroscopically obvious Crohn's disease can undergo resolution, unrelated to treatment. It is common experience that colonic Crohn's disease can progress very rapidly when assessed by serial radiological or endoscopic evaluation. Cases 1 and 2 show that patients with no macroscopic evidence of disease at first laparotomy may develop severe disease over a relatively short time (two years and nine months).

Resolution is much less clearly documented than progression, though there is some evidence for this. Case 3 shows that the macroscopic appearances of Crohn's disease may regress, in that the first two laparotomies showed typical features of the disease, but the third showed normal bowel. Resolution could be related to medical treatment, but the striking feature of these cases was that symptoms did not resolve, and laparotomy in each case was undertaken because of failure to respond (symptomatically) to medical treatment.

It may be concluded that normal laparotomy findings do not necessarily exclude a diagnosis of intestinal Crohn's disease, or the presence of sufficient disease activity to be symptomatic, and that relatively rapid macroscopic changes may occur, both progression and resolution. It is interesting to sepculate on the cause of the symptoms in these cases with macroscopically normal bowel, where it is unlikely that mechanical obstruction played a part. Intense muscle spasm is a conspicuous feature of small bowel Crohn's disease - perhaps mucosal inflammation can give enough muscle contraction to cause ischaemic pain. The superficial nature of the histological changes in the resected cases is in contrast to the extensive transmural inflammation typical of Crohn's disease, and could explain the lack of palpable thickening of the bowel wall.

The major problem posed by these cases relates to management, since four of six patients continued with clinical symptoms of considerable severity. One possibility would be to proceed with resection of the clinically and radiologically suspicious bowel, despite its normal macroscopic appearance. This would create the problem of how to determine the resection margins, and it should be noted that in two of the cases discussed recurrence occurred in an area of the bowel not previously under suspicion. However, from our experience we have concluded that a firm decision regarding resection should be made before laparotomy, and that the patient's interests will best be served by resection, even in the presence of macroscopically normal bowel. Resection should concentrate on the area of radiological abnormality, and the limits determined by inspecting the mucosa of the resected bowel before anastomosis. Patients with persisting symptoms after a previous negative laparotomy should be reassessed carefully on the basis that they are very likely to have appreciable disease.

1 Crohn BB, Ginsberg L,'Oppenheimer GD. Regional ileitis: a clinical and pathological entity. $\mathcal{f} A M A$ 1932; 99: 1323-9.

Lindner AE, Marshak RH, Wolf BS, Janowitz HD. Granulomatous colitis. N Engl F Med 1963; 296: 379-85.

Brooke BN. Granulomatous diseases of the intestine. Lancet 1959; ii: 745-9.

4 Warren S, Sommers SC. Pathology of regional ileitis and ulcerative colitis. FAMA 1954; 154: 189-93.

5 Gump FE, Lepore M, Barker HG. A revised concept of acute regional enteritis. Ann Surg 1967; 166: 942-6.

6 Weakley FL, Turnbull RB. Recognition of regional ileitis in the operating room. Dis Colon Rectum 1971; 14: 17-23.

Lockhart-Mummery HE, Morson BC. Crohn's disease of the large intestine. Gut 1964; 5: 493-509.

8 Lockhart-Mummery HE, Morson BC. Crohn's disease of the large intestine and its distinction from ulcerative colitis. Gu 1960; 1: 87-105.

9 Cornes JS, Stecher M. Primary Crohn's disease of the colon and rectum. Gut 1961; 2: 189-210.

10 Cook MG, Dixon MF. An analysis of the reliability of detection and diagnosis value of various pathological features in Crohn's disease and ulcerative colitis. Gut 1973; 14: $255-62$.

11 Morson BC, Dawson IMP, Day DW, Jass JR, Price AB Williams GT, et al. Morson and Dawson's gastrointestina pathology. 3rd ed. Oxford: Blackwell Scientific, 1990: 267. 